\title{
Informes
}

\section{México: educación pública y formación de un investigador en ciencias sociales*}

\author{
Gustavo Garza**
}

En este texto se reproduce la versión completa del discurso pronunciado por Gustavo Garza en la sesión solemne del H. Consejo Universitario de la Benemérita Universidad Autónoma de Puebla, realizada el 20 de noviembre del 2014, en la cual se le otorgó el grado de Doctor Honoris Causa. Su argumento central es que los científicos sociales no surgen por mera genética, vocación o virtuosismo, sino que son producto de las instituciones públicas de investigación universitaria, dentro del contexto social, económico y político en que se desenvuelven, además de ciertos atributos subjetivos. Utiliza su ciclo de vida escolar y de investigador para demostrar dicho planteamiento, siguiendo cronológicamente sus estudios y publicaciones principales: Facultad de Economía, Universidad Autónoma de Nuevo León, alma mater primigenia (1962-1967); El Colegio de México, segunda alma mater (1967-1969); formación autodidacta en economía urbana (1970-1976); Universidad de Cambridge, tercera alma mater (1972-1973); investigador individual, UNAM, cuarta alma mater (1975-1985); dos décadas de libros convencionales (1985-2005); hacia una escuela de economía política urbana (20052014); BUAP, quinta alma mater. Concluye que como una medida para revertir la grave violencia criminal que socava y amenaza con devastar a México, se debe reforzar la calidad académica de las universidades públicas, formando más y mejores estudiantes e investigadores.

\section{Mexico: Public Education and Training of a Social Science Researcher}

This text reproduces the full version of the lecture delivered by Gustavo Garza at the solemn session of the University Council of the Autonomous University of Puebla, on November 20, 2014, when he was awarded an honorary doctorate. Dr. Garza's central argument is that social scientists do not simply emerge as a result of genetics, vocation or virtuosity but are the product of public university research institutions within the social,

* Versión original completa del discurso presentado por Gustavo Garza en la sesión solemne del H. Consejo Universitario, cuando se le otorgó el grado de Doctor Honoris Causa por la Benemérita Universidad Autónoma de Puebla, el 20 de noviembre de 2014. En la ceremonia se presentó una versión resumida de 19 minutos.

** Profesor investigador del Centro de Estudios Demográficos, Urbanos y Ambientales de El Colegio de México. Dirección postal: Camino al Ajusco 20, Col. Pedregal de Santa Teresa, C.P. 14200, México, D.F., México. Correo electrónico: <ggarza@colmex.mx). 
economic and political context in which they operate, in addition to certain subjective attributes. He uses his life at university and as a researcher to demonstrate this approach, chronologically charting his studies and major publications: Faculty of Economics, Universidad Autónoma de Nuevo León, first alma mater (1962-1967); El Colegio de Mexico, second alma mater (1967-1969); self-taught in urban economics (1970-1976); University of Cambridge, third alma mater (1972-1973); individual researcher, UNAM, fourth alma mater (1975-1985); two decades of conventional books (1985-2005); towards a school of urban political economy (2005-2014); BUAP, fifth alma mater. Dr. Garza concludes by saying that as a measure to reverse the serious criminal violence undermining and threatening to devastate Mexico, the academic quality of public universities should be strengthened to train more and better students and researchers.

El producto interno bruto (PIB) de México aumentó $6.5 \%$ anual durante los años sesenta del "milagro económico", casi duplicándose cada decenio. En ese tiempo terminar una carrera universitaria era garantía de ascenso social. Empero, desde la preparatoria yo había cursado las materias de Filosofía y de Economía Política, por lo que era lo suficientemente consciente de las características de México, que eran y continúan siendo las de un país esencialmente subdesarrollado, política, social y económicamente.

\section{Facultad de Economía, UANL: alma mater primigenia (1962-1967)}

En septiembre de 1962 tuve la fortuna de ser elegido entre cerca de 300 candidatos para estudiar en la recién fundada Facultad de Economía de la Universidad Autónoma de Nuevo León (UANL). La institución era dirigida por Consuelo Meyer, comisionada por el Banco de México para formar una institución con elevados estándares académicos. Entre los maestros extranjeros que impartían las clases anuales estaba Dominique Hachett, francés (Introducción a la Economía); Leoncio Durandeau, chileno (Teoría Económica); Ingolff Otto, alemán (Moneda y Banca); Eduardo Ruiz, norteamericano (Historia Económica de América Latina); Elizabeth Jelin y Jorge Balán, argentinos (Ciencias Sociales). Los maestros mexicanos impartían Historia de las Doctrinas Económicas, Comercio Internacional, Finanzas Públicas, Mercadotecnia, Economía Agrícola y Programación Lineal. Además, había jóvenes docentes mexicanos egresados de la facultad que habían cursado sus posgrados en el extranjero, tal es el caso de Jesús Puente Leyva (Desarrollo Económico) y el de Ernesto Bolaños (Geografía Económica). Existían, por supuesto, maestros encargados de cursos técnico-instru- 
mentales, como Matemáticas para Economistas, Contabilidad Nacional y Estadísticas Económicas.

Cabe destacar que se impartían dos cursos anuales titulados Evolución de la Civilización Contemporánea, I y II. En ellos se estudiaba el devenir del conocimiento desde la edad antigua con la aparición del pensamiento racional iniciado por los griegos (Parménides, Diógenes, Pitágoras, Sócrates, Platón, Aristóteles) y los romanos (Cicerón, Séneca, Catón); la edad media con su mística filosófico-religiosa (Averroes, San Agustín, Santo Tomás de Aquino, Tomás Moro, Calvino y Erasmo de Róterdam); y la época moderna con el despertar del renacimiento (Descartes, Copérnico, Galileo, Hobbes, Pascal, Spinoza, Locke, Newton, Leibnitz, Voltaire, Hume, Russeau, Montesquieu, Diderot, Kant y Hegel), hasta llegar al positivismo y marxismo del siglo XIX y primera mitad del XX (Schopenhauer, Goethe, Darwin, Malthus, Marx, Engels, Nietzsche y Einstein).

En mi caso, la capacidad de polemizar, reflexionar con cierta profundidad y trabajar con la dedicación que debe poseer un académico, hasta donde tenga algo de ello, tuvo su formación originaria entre 1962 y 1967, cuando realicé mis estudios de licenciatura en la Facultad de Economía de la UANL, mi alma mater primigenia.

La UANL es una institución estatal. Había estudiado la primaria, secundaria y preparatoria en escuelas gubernamentales, por lo que resultaba ser un joven universitario producto de la educación pública en México, cuyo significativo desarrollo constituyó uno de los grandes logros de la Revolución Mexicana de 1910.

En 1960 México tenía una población de 35 millones de habitantes, de los cuales 13 millones eran urbanos (representaban 39\% del total). El país permanecía esencialmente rural con islotes urbanos que experimentaban un acelerado proceso de industrialización, entre los que sobresalían cinco: Ciudad de México, Guadalajara, Monterrey, Puebla y Torreón. Era entonces imposible prever que tres años después de terminar la universidad, en 1967, me iba a iniciar como un joven investigador en un proyecto titulado "El proceso de urbanización en México", coordinado por Luis Unikel.

El Colegio de México: segunda alma mater (1967-1969)

A principios de 1967 solicité entrar a la maestría en Economía en el Centro de Estudios Económicos y Demográficos (CEED) de El Colegio 
de México, cuando cursaba el quinto año de la carrera. Supe de la existencia de la institución pues uno de sus profesores fue a Monterrey a invitarnos a presentar la solicitud para ingresar a la maestría. Me respondieron que la aceptación dependería de si terminaba con buenas notas dicho año escolar. Por ello, para no descuidar los estudios, decidí renunciar a mi empleo como encargado del departamento de reserva legal del Banco General de Monterrey. En julio, al culminar los estudios, envié mis calificaciones y en agosto me llegó un telegrama: Felicidades, aceptado Maestría Economía, clases inician 4 de septiembre 9:00. Se ofrecía una beca mensual de \$1500.00, de la cual, como buen regiomontano, empecé a ahorrar $20 \%$ para enfrentar contingencias y sufragar algunos viajes a Monterrey.

Transformado en migrante interno por motivos escolares, llegué a la capital del país el domingo 3 de septiembre en la noche. Sólo había estado una vez en la urbe, pero me trasladé en autobús a la colonia Roma, calle Guanajuato 125, donde se encontraba El Colegio de México. Me percaté inmediatamente que se trataba de una institución de excelencia en investigación y docencia. Su presidente era Víctor L. Urquidi, uno de los economistas más distinguidos del país. Lo conocía como autor importante, pues en el curso de desarrollo económico en la facultad había leído su libro Viabilidad económica de América Latina (México, FCE, 1962).

Creía que el tiempo dedicado al estudio como alumno de la Facultad de Economía era lo máximo posible, pero me equivocaba. El Colegio exigía más, además de que la mayor parte de las lecturas estaban en inglés, lo cual representaba para mí una desventaja pues me exigía más tiempo. La constante amenaza de ser suspendido del programa al reprobar cualquier clase o tener menos de 8 de promedio era otra fuente de estrés. Empecé a economizar tiempos perdidos y a maximizar los de estudio. Después de un tropezón inicial en un primer examen de matemáticas en el que obtuve 5, nota que representaba $50 \%$ de la calificación final, logré sacar 10 en un segundo examen para promediar 7.5 y aprobarla. En ese primer semestre el curso de Teoría Económica I lo impartió Carlos Tello. Del segundo semestre cabe destacar Teoría Económica II, con Sergio Ghigliazza, y Comercio Internacional, con Gerardo Bueno. Del tercer semestre se puede mencionar Teoría Monetaria y Fiscal, con Jesús Silva Herzog, Teoría del Desarrollo Económico, con Saúl Trejo, y Sociología del Desarrollo, con Claudio Stern. En el último semestre se impartió Programación Económica, por Francisco Javier Alejo y el Seminario de Desarrollo y 


\section{INFORMES}

Planeación, por Leopoldo Solís. Como trabajo de investigación empecé a escribir sobre la evolución del sistema bancario en México.

Tal fue mi formación en la Maestría de Economía, y su realización contribuyó a reforzar la disciplina por el trabajo y el conocimiento de la teoría económica y temas consustanciales a la formación de un economista, principalmente sobre los determinantes del desarrollo económico de las naciones.

El Colegio de México es un organismo descentralizado que depende fundamentalmente del presupuesto federal. Mi segunda alma mater, por ende, pertenece al Estado, elevando mi deuda con el sistema educativo público financiado por el pueblo de México.

En 1970 México tenía una población de 48 millones de habitantes, de los cuales $53 \%$ era rural y $47 \%$ se distribuía en 176 ciudades. Persistía el idiosincrático perfil campesino del país de la primera mitad del siglo Xx, pero la Ciudad de México absorbía 8.6 millones de habitantes, mientras que Guadalajara y Monterrey 1.5 y 1.2 millones, siguiendo Puebla con 629 mil y León con 470 mil habitantes.

En la transición de México hacia una nación más urbanizada, al terminar la maestría empecé a buscar empleo en instituciones bancarias, tema de mi tesis. Sin embargo, me enteré que en El Colegio "andaban buscando un economista" para trabajar en un proyecto de investigación sobre el proceso de urbanización en México. Aunque mi ignorancia sobre el tema era total, me entrevisté con Luis Unikel, coordinador del proyecto, quien me explicó que requería de un economista para analizar las funciones económicas de las ciudades, su estructura de consumo, finanzas y desigualdades de ingreso. No recuerdo si le comenté mi total desconocimiento de las cuestiones económicas de las ciudades, pues incluso no sabía de la existencia de la especialidad en economía urbana. Supongo que no dije nada, pues ¡me aceptó para trabajar en el proyecto!

\section{Formación autodidacta en economía urbana (1970-1976)}

El 5 de enero de 1970, a los 24 años, el joven que había emigrado a la Ciudad de México para estudiar un posgrado se inició como investigador por proyecto en El Colegio de México, su segunda alma mater.

La disciplina de trabajo y el pensamiento conceptual que había adquirido en mis estudios me permitieron iniciarme como especialista en un tema que me era absolutamente ajeno. Actualmente, 
después de 40 años de escribir extensivamente sobre economía urbana, me es posible afirmar que algo significativo puedo decir al respecto, pero queda siempre mucho por aprender. Empecé por estudiar un documento de alrededor de 200 páginas titulado "El proceso de urbanización en México: anteproyecto de investigación", que se había realizado en 1966 para guiar la realización del trabajo. Mi primera tarea fue determinar y analizar la especialización económica de las 37 principales ciudades de México entre 1940 y 1970, labor que realicé en el primer semestre de 1970. Sus resultados, publicados en forma de artículo en coautoría con el coordinador del proyecto, constituyeron la primera publicación de mi carrera, siendo por ello especialmente significativa:

Unikel, Luis y Gustavo Garza (1971), "Una clasificación funcional de las principales ciudades de México”, Demografía y Economía, vol. 5, núm. 3, pp. 329-359. Disponible en: <jstor.org/stable/40601940>.

La culminación de mi etapa de investigador dentro del proyecto de Luis Unikel fue en diciembre de 1974, cuando se terminó el manuscrito completo del trabajo, que se tituló "El desarrollo urbano de México. Diagnóstico e implicaciones futuras". Por iniciativa del presidente de El Colegio de México, Víctor L. Urquidi, el documento se presentó al Premio Nacional de Economía, Banamex, en 1975, y obtuvo el primer lugar.

Su versión en forma de libro apareció en 1976, y en ese mismo año se fundó el área de Estudios Urbanos dentro del CEED, con un programa de Maestría en Desarrollo Urbano y una serie de proyectos de investigación. Los alumnos de las primeras promociones bautizaron al libro como "la biblia" del desarrollo urbano, pero los estudiantes actuales prácticamente no lo conocen pues la información que incluye es hasta 1970, y el tema de la urbanización se estudia en libros y artículos más recientes. Permanece, empero, como un libro de consulta clásico para los investigadores de la especialidad.

Sea como fuere, fui verdaderamente afortunado en iniciar mi carrera de investigador trabajando durante cuatro años en uno de los proyectos más rigurosos e importantes en cuestiones urbanas y regionales que se han realizado en México. El aprendizaje logrado en ese tiempo, en buena medida autodidacta, constituyó un verdadero doctorado de investigación en estudios urbanos dentro de mi segunda alma mater. Como me brindó también la oportunidad de formarme 


\section{INFORMES}

como investigador profesional, mi reconocimiento, agradecimiento y deuda a una distinguida institución pública de investigación y docencia es, por lo tanto, doble.

Generalizando mi caso, se evidencia incuestionablemente la función crucial que desempeñan las instituciones de educación pública universitaria en la formación académica y profesional de los cuadros de científicos en México, tanto en las ciencias naturales como en las sociales.

\section{Universidad de Cambridge, 1972-1973: tercera alma mater}

El University College London, de la Universidad de Londres, ofrecía un diplomado anual sobre el proceso de urbanización. En 1972 fui a cursarlo, pero estando allá se suspendió y me dieron la opción de asistir a un curso sobre Desarrollo Económico en la Universidad de Cambridge, lo cual acepté. Al terminar los exámenes, unos cuantos alumnos obtuvimos el diploma correspondiente y mi tutor me comunicó que me aceptaban como doctorando. Por una serie de razones decliné y regresé a México, donde continué trabajando un año y medio en el proyecto del proceso de urbanización hasta que, felizmente, lo culminamos.

La tesis que presenté en Cambridge fue:

Garza, Gustavo (1973), "Towards a National Strategy of Industrial Estates in Mexico", tesis de diplomado, Cambridge, Wolfson College, Cambridge University.

El trabajo fue la base conceptual para, casi 20 años después, escribir el libro:

Garza, Gustavo (1992), Desconcentración, tecnología y localización industrial en México. Los parques y ciudades industriales, 1953-1988, 1aㅡ ed. [1 000 ejemplares], México, El Colegio de México, 457 págs. ISBN: 968-120-499-9.

Ello ejemplifica la filosofía que he aplicado a lo largo de mi carrera: todo lo que escribes que consideres no publicable, puede ser desarrollado y utilizado en el futuro para un proyecto de mayor envergadura.

La universidad de Cambridge, fundada oficialmente en 1231 mediante un decreto del rey Enrique III, es una institución de investigación 
pública. Está constituida por seis escuelas, más 150 departamentos y facultades, así como por 31 colegios autónomos con patrimonio propio. Durante mi estancia tuve una beca del Conacyt complementada por otra del British Council. De lo anterior se deriva que el aprendizaje recibido, en esta oportunidad en la Universidad de Cambridge -una de las más antiguas y prestigiadas del mundo-, también provenga de un organismo público, que se convirtió en la tercera alma mater de mi vida escolar.

En 1991 fui invitado con pago salarial por el Centre of Latin American Studies de la Universidad de Cambridge, para el periodo abriljulio. En esa oportunidad principié la lectura y recopilación sistemática de bibliografía especializada en el sector servicios y sus peculiaridades territoriales, tarea que estuve haciendo de manera intermitente durante 15 años, antes de iniciar en 2005 la investigación sobre la organización espacial del sector servicios en México.

\section{Investigador individual: UNAM, cuarta alma mater (1975-1985)}

A partir de la culminación de "El desarrollo urbano de México", en 1975 se me otorgó la planta de profesor investigador de El Colegio de México e inicié mi carrera docente en la Maestría en Desarrollo Urbano como investigador con sus propios proyectos. ¡Fue el momento de la verdad!

El Banco Interamericano de Desarrollo (BID) financiaba el pago salarial de los maestros de dicha maestría y la beca de los alumnos, en ambos casos a extranjeros. El BID solicitó al Centro de Estudios Económicos y Demográficos que participase con una ponencia sobre la situación de la vivienda en México en un encuentro latinoamericano que se realizaría en Washington. La dirección del Centro conminó a Martha Schteingart y a un servidor a elaborar el estudio. Nuevamente me encontraba ante un tema nunca antes investigado por mí, pero nos pusimos a trabajar arduamente y, más que el breve documento solicitado, elaboramos un libro que fue publicado como:

Garza, Gustavo y Martha Schteingart (1978), La acción habitacional del Estado en México, 1a ed. [3 000 ejemplares], México, Centro de Estudios Económicos y Demográficos, El Colegio de México, 245 págs. 
Este segundo libro demuestra que la disciplina de trabajo y una buena formación en ciencias sociales permiten abordar cualquier tema de la especialidad, sin importar que no se haya estudiado antes.

En 1979 realicé mi primera investigación individual que, siendo un trabajo relativamente modesto, establecía un planteamiento concreto para implementar una estrategia de desarrollo económico y urbano para México:

Garza, Gustavo (1980), Industrialización de las principales ciudades de México, $1^{\underline{a}}$ ed. [3 000 ejemplares], México, El Colegio de México, 155 págs. ISBN: 968-12-0065-9.

Específicamente seleccioné tres ciudades para que constituyeran casos especiales donde centrar las políticas de infraestructura y promoción económica, de tal suerte que conformaran los motores del desarrollo y de la descentralización industrial. No obstante que la investigación se realizó con apoyo financiero de la antigua SAHOP (Secretaría de Asentamientos Humanos y Obras Públicas), al parecer nadie de dicha dependencia lo leyó. Una década después me enteré que en ese año, en su séptimo plan quinquenal, la República Popular China había establecido sus primeras zonas económicas especiales, esto es, la misma idea. Ellos las desarrollaron sistemáticamente hasta llegar a tener polos mundiales como Shenzhen, en la provincia de Guangdong, y el megaproyecto de Pudong, en Shanghái. Los resultados están a la vista, pues China ha crecido $10 \%$ anual y México 2.5\%. China requiere siete años para duplicar su PIB y México treinta.

¡Mejor dedícate a la investigación teórica y olvídate de intentar imprimir un rigor científico al empirismo gubernamental!, me dije. El ánimo no decayó, pero se reorientó.

En 1977 inicié mis estudios de doctorado en la Facultad de Economía de la Universidad Nacional Autónoma de México (UNAM), siguiendo como investigador en El Colegio de México. En agosto de 1980 obtuve mi candidatura presentando un proyecto de investigación y algunos avances sobre la evolución y los determinantes de la concentración industrial en la Ciudad de México. Dos años después entregué la versión final de la tesis y el 9 de febrero de 1983 se me otorgó el grado, además de la medalla Gabino Barreda. Posteriormente la tesis se publicó en forma de libro como: 
Garza, Gustavo (1985), El proceso de industrialización de la Ciudad de México, 1821-1970, 1ํㅡㄹ ed. [2 000 ejemplares], México, El Colegio de México, 647 págs. ISBN: 968-120-308-9.

Desde mi perspectiva, la obra constituye uno de los trabajos de mayor envergadura teórica, conceptual y estadística que he realizado. Se logró identificar el principal determinante de la concentración industrial en el espacio mediante la categoría histórica denominada condiciones generales de la producción (CGP) -infraestructura y equipamiento-, cuyo valor dividido entre el capital fijo privado constituye lo que bauticé como coeficiente de la composición interna del capital constante fijo. Su mayor valor en la Ciudad de México permite que sus empresas tengan tasas de ganancia más elevadas. El planteamiento teórico fue validado con un andamiaje estadístico desarrollado mediante una compleja metodología que hizo posible cuantificar las tasas de ganancia y el coeficiente señalado. Ello permitió evidenciar que las ciencias sociales, al igual que las naturales, pueden ser de carácter nomotético, esto es, son capaces de formular leyes del comportamiento de los fenómenos del mundo real. Ello se ajusta cabalmente al artículo $16^{\circ}$ de la Ley Orgánica de la Benemérita Universidad Autónoma de Puebla (BUAP), que apunta: "La investigación es un trabajo sistemático y creativo realizado con el fin de avanzar en la frontera del conocimiento sobre la naturaleza, el hombre, la cultura y la sociedad".

Paralelamente la UNAM, como la principal universidad de la nación, me permitió consolidar mi formación profesional y constituyó mi cuarta alma mater. Dado su carácter de universidad pública por excelencia, se ratifica mi formación en el seno de las instituciones educativas pertenecientes al Estado mexicano.

En reciprocidad, y como muestra de mi sincera gratitud con ellas, he realizado mi mayor esfuerzo en corresponder a la sociedad mexicana con obras que reflejen el más elevado rigor científico que me ha permitido alcanzar mi capacidad intelectual y sistematicidad laboral.

\section{Dos décadas de libros convencionales (1985-2005)}

El producto interno bruto de México experimentó una tasa de $6.6 \%$ en los setenta, en las postrimerías del denominado "milagro económico". En los ochenta, después del crac financiero de 1982, terminó la época del crecimiento acelerado e inició la "década perdida", cuando 


\section{INFORMES}

el PIB decreció ligeramente entre 1982 y 1988. A partir de entonces México se inserta en las políticas neoliberales dictadas por el consenso de Washington que gradualmente reducen la posición rectora del Estado mediante la privatización de las empresas públicas, hasta llegar en buena medida a las dos últimas en 2014: Pemex y la CFE.

Dicha estrategia tuvo su epicentro en Estados Unidos e Inglaterra, pero se impone a toda la Unión Europea y a algunos países latinoamericanos y asiáticos. Ello impactó de manera significativa a los cuerpos de investigación pues las exigencias macroeconómicas se acompañan con la imposición de enfoques metodológicos y temas de investigación acordes con la corriente anglosajona hegemónica. Lo más notable para nuestros propósitos es la desaparición de la escuela francesa de sociología urbana de corte marxista y la generalización de enfoques técnicos de corte neoclásico y estadístico: la economía, sociología y ciencia política se trasforman en ramas de la econometría. Se pretende con ello disimular las crisis recurrentes, la acentuación de las desigualdades sociales y la inviabilidad histórica del esquema neoliberal como programa de desarrollo viable y sustentable.

En forma intuitiva y por la atmósfera prevaleciente en casi todos los ámbitos, me orienté a trabajar en lo que denomino obras convencionales, que aunque son significativas dentro del estado de conocimiento en México, ninguna alcanza el umbral nomotético del trabajo anterior. De los 13 libros publicados en esos 20 años a razón de uno cada 18 meses, convendría destacar los siguientes, de autoría o coautoría, con uno muy especial en el que fungí como coordinador:

Garza, Gustavo y Jaime Sobrino (1989), México: industrialización periférica en el sistema de ciudades de Sinaloa, 1960-1992, 1aㅡ ed. [1000 ejemplares], México, El Colegio de México, 343 págs. ISBN: 968-120-436-0.

Garza, Gustavo (1992), Desconcentración, tecnología y localización industrial en México. Los parques y ciudades industriales, 1953-1988, 1ª ed. [1000 ejemplares], México, El Colegio de México, 457 págs. ISBN: 968-120-499-9.

Garza, Gustavo y Salvador Rivera (1994), Dinámica macroeconómica de las ciudades en México, 2ª ed. [500 ejemplares], México, INEGI / IIS-UNAM / El Colegio de México, 120 págs. ISBN: 970-130-271-0.

Garza, Gustavo (1996), Cincuenta años de investigación urbana y regional en México, 1940-1991, 1aㅡ ed. [1000 ejemplares], México, El Colegio de México, 325 págs. ISBN: 968-12-0689-4. 
Garza, Gustavo (1998), La gestión municipal en el Área Metropolitana de Monterrey, 1989-1994, México, Miguel Ángel Porrúa / IIS-UnAM / El Colegio de México, 476 págs. ISBN: 968-842-814-0.

Garza, Gustavo (coord.) (2000), La Ciudad de México en el fin del segundo milenio, $1^{\text {a }}$ ed. [3000 ejemplares], México, Gobierno del Distrito Federal / El Colegio de México, 768 págs. ISBN: 968-120-996-6.

Garza, Gustavo (2003), La urbanización de México en el siglo XX, México, El Colegio de México, 208 págs. ISBN: 968-12-1107-3 [reimpreso en 2005].

Garza, Gustavo, Pierre Filion y Gary Sands (2003), Politicas urbanas en grandes metrópolis: Detroit, Monterrey y Toronto, México, El Colegio de México, 402 págs. ISBN: 968-12-1064-6.

A los libros se les pueden sumar 107 artículos y capítulos publicados en esos 20 años, promediando 5.3 anuales, aunque un buen número de ellos forman parte de las publicaciones realizadas en calidad de coordinador, las cuales no han sido mencionadas.

Cabría resaltar, en primer lugar, las varias temáticas de los anteriores trabajos, que van desde el análisis macroeconómico de las ciudades, la evaluación rigurosa de las políticas urbanas y regionales, el proceso de urbanización, la gestión de las ciudades y el estudio del estado de conocimiento en la disciplina.

En segundo lugar, parece pertinente justificar por qué se incluye un libro que coordiné, es decir, La Ciudad de México en el fin del segundo milenio. Se trata de un trabajo enciclopédico que incorpora la dimensión histórica, geográfica, urbana, sociodemográfica, ecológica, de gestión, política, estructuración del suelo y prospectiva del Área Metropolitana de la Ciudad de México, de manera analítica, estadística, cartográfica y fotográfica. Para ello se coordinó a casi un centenar de especialistas en todos los anteriores temas, cuyos trabajos fueron presentados en una serie de monografías con uniformidad estilística, lo cual me significó revisar y corregir el total de los trabajos. Sobre la obra se ha afirmado que: "Este libro es la más importante investigación hecha sobre el desarrollo urbano de nuestra ciudad" (Antonio Toca, Excélsior, 01/06/2013).

La posibilidad de realizar tal tarea, al igual que abordar diferentes temáticas de la disciplina urbana en las investigaciones, se debe básicamente a la formación multidisciplinaria obtenida de mis cuatro alma 
mater, que representan otras tantas instituciones de educación pública, principalmente de México.

\section{Hacia una escuela de economía política urbana (2005-2014)}

La crisis financiera de las hipotecas subprime que irrumpe en agosto de 2007 en Estados Unidos y se internacionaliza en 2008 y 2009 con el rescate de múltiples bancos en situación de quiebra, lejos de ser resuelta, entra en una tercera fase más aguda en Europa por el estallido de la crisis de deuda griega a inicios de 2010. La nueva situación se intenta paliar con masivos programas de rescate del Banco Central Europeo y la exigencia de un riguroso programa de austeridad que ha implicado cinco años de crecimiento negativo en Grecia. A ella se agregaron Irlanda y Portugal, y en España, Bélgica, Italia y Francia se extendieron los programas de ajuste, condenando a esos países a un estancamiento económico de largo plazo. Se acepta generalmente que se trata de la crisis capitalista más grave desde la posguerra, pero se debate ampliamente su naturaleza: ¿sistémica o salvable?

Dentro de estas turbulentas aguas, el buque de los BRICS (Brasil, Rusia, India, China y Sudáfrica) sigue navegando, principalmente por la asombrosa dinámica de China, que en 2014 se ha transformado en la primera economía del planeta, según el PIB medido por paridad de poder adquisitivo. El país se ha desarrollado siguiendo una estrategia de planificación central y actualmente está elaborando su $13^{\circ}$ Plan Quinquenal, 2015-2020. Para ello tiene siete comisiones que trabajan desde hace dos años, una de las cuales se encarga de estudiar la función de las ciudades y la del sector terciario dentro de la estrategia futura de desarrollo.

En el nuevo contexto geopolítico, y visualizando la trascendencia de la revolución terciaria que los principales países del mundo estaban experimentando desde la segunda mitad del siglo $\mathrm{XX}$, reorienté mis tópicos de estudio hacia la organización espacial del sector comercio y servicios en México, lo cual me permitió retornar al enfoque de economía política urbana. Se trata, idealmente, de impulsar el surgimiento de una escuela de pensamiento en esa rama cognoscitiva. Este paradigma ha sido reivindicado por el reciente libro de Thomas Piketty, El capital en el siglo XXI (2014), que ha causado cierto revuelo entre los economistas neoclásicos anglosajones, entre otras cosas por concluir que: "Veo a la economía como una subdisciplina de las ciencias socia- 
les $[\ldots]$ no me gusta la expresión ciencia económica, prefiero mucho más la de economía política" ("Conclusiones", versión digital). Se comparte la certeza de la gran relevancia de esta rama de conocimiento para el desarrollo nomotético de las ciencias sociales en el mundo. En el caso de México constituirá una herramienta analítica fundamental para diseñar nuevas estrategias con objeto de salir de la crisis crónica de las últimas tres décadas, e instrumentarlas cuando la situación política lo permita.

El impulso a la investigación sobre la organización espacial del sector terciario se logró ampliamente al publicarse, entre 2006 y 2011, una pentalogía sobre la estructura, la participación, la dinámica y la organización espacial del sector comercio y servicios en México, el cual contribuye con más de $70 \%$ del PIB nacional. Los cinco libros cubren las 32 entidades federativas y analizan 100 de sus principales ciudades para el periodo de 1980 a 2003. En todos los casos de realizó el mismo ejercicio estadístico de comparabilidad de los censos económicos, así como el ajuste del PIB terciario censal para hacerlo equiparable con las cuentas nacionales y, con ello, permitir su estudio diacrónico. Los libros realizados, con la participación de un grupo de colegas y algunos estudiantes de posgrado, son:

Garza, Gustavo (coord.) (2006), La organización espacial del sector servicios en México, México, El Colegio de México, 555 págs. ISBN: 968-12-1249-5.

Garza, Gustavo (2008), Macroeconomía del sector servicios en la Ciudad de México, 1960-2003, México, El Colegio de México, 593 págs. ISBN: 978-96812-1380-0.

Garza, Gustavo y Jaime Sobrino (coords.) (2009), Evolución del sector servicios en ciudades y regiones de México, México, El Colegio de México, 875 págs. ISBN: 978-607-462-034-4.

Garza, Gustavo (coord.) (2010), Geografía del sector servicios en el norte de México, $1^{\mathrm{a}}$ ed., México, El Colegio de México / Universidad Autónoma de Coahuila, 658 págs. ISBN: 978-607-462-049-8.

Garza, Gustavo (coord.) (2011), Visión comprensiva de la distribución territorial del sector servicios en México, 1ํㅡㄹ ed., México, El Colegio de México, 880 págs. ISBN: 978-607-462-223-2. 
El capítulo XII del último libro presenta una visión totalizadora del proceso de servicialización según 8 regiones, 32 entidades federativas y 100 ciudades, para el periodo de 1980 a 2003. Con la información del PIB terciario en los tres niveles espaciales anteriores se diseñó un ejercicio estadístico para validar empíricamente una teoría unificada espacio-sectorial del desarrollo económico, propuesta en el capítulo. Se concluyó que las condiciones generales de la producción (CGP), como categoría histórica, constituyen un capital socializado indispensable para la acumulación ampliada del capital de las empresas privadas y, por ende, el vínculo entre el desarrollo económico y su organización territorial. Se establece que "es preciso continuar estudiando la naturaleza de la función de las CGP en el desarrollo económico de las ciudades y regiones, cuestión de cardinal importancia para la economía política urbana" (Garza, 2011: 838). Se intenta trascender el análisis empírico e impulsar propuestas teórico-metodológicas de corte nomotético, como en la física, la química y la biología.

Para ello sería necesario promover el desarrollo de una escuela de pensamiento de economía política urbana, y hacia ese propósito dirigí la parte más reciente de mis trabajos de investigación. Para avanzar en esa dirección se propuso la realización de una trilogía que tuviera como propósito general validar estadísticamente la teoría unificada espacio-sectorial del desarrollo económico propuesta en la publicación de 2011. En el libro primero se plantea la existencia del binomio condiciones y servicios generales de la producción, que se conceptualiza como parte integral de la teoría del capital, a la que se le imprime un carácter tridimensional; se agrega el desarrollo histórico de las CGP en el mundo; y se incorpora un capítulo que presenta la definición, tipología y características de dicho binomio. La referencia bibliográfica del libro es:

Garza, Gustavo (2013), Teoría de las condiciones y los servicios generales de la producción, México, El Colegio de México, 382 págs. ISBN: 978-607462-450-2.

El libro segundo valida parcialmente dicha teoría cuantificando el valor del capital constante de los medios de producción socializados de la urbe: sistema hidráulico, servicio eléctrico, dotación de hidrocarburos, red vial, metro-metrobús y telecomunicaciones. La cantidad alcanzada para 2008 es de 1.2 millones de millones de pesos, mientras el capital fijo privado representa 1.3, resultando un coeficiente de 0.9 . 
De esta suerte, por cada peso invertido en las empresas se requieren 90 centavos de capital socializado. La ficha bibliográfica es:

Garza, Gustavo (coord.) (2014), Valor de los medios de producción socializados en la Ciudad de México, México, El Colegio de México, 326 págs. ISBN: 978607-462-596-7.

Finalmente, en el libro tercero se cuantifica el valor de los siguientes medios de consumo colectivos de la metrópoli: inventario habitacional, sistema educativo, equipamiento de salud, mobiliario cultural, edificios gubernamentales y áreas verdes. El valor alcanzado por estos renglones, exceptuando a las viviendas, junto con el correspondiente a los medios de producción socializados del libro segundo, es de 2.5 millones de millones de pesos, mientras el capital fijo privado se mantiene en 1.3, resultando un coeficiente de 1.9. Ello implica que por cada peso de capital privado se requieren 1.90 pesos de capital socializado de infraestructura y equipamiento, esto es, condiciones generales de la producción. El libro se titula:

Garza, Gustavo (2015), Valor de los medios de consumo colectivos en la Ciudad de México, México, El Colegio de México, 628 págs.

La validación estadística de la teoría establecida representa un avance en la investigación de la naturaleza de la ciudad como fuerza productiva monumental, el cual contribuirá indudablemente a impulsar el progreso conceptual de la economía política urbana.

Lo anterior describe las publicaciones centrales de mis labores de investigación, a lo cual habría que agregar la satisfacción de tener alrededor de cuatro décadas de ser maestro en algunas clases y seminarios en la Maestría de Estudios Urbanos en el CEDUA de El Colegio de México, así como en otras instituciones. Actualmente imparto Economía Política Urbana en dicha maestría y Metodología de las Ciencias Sociales en el nuevo Doctorado de Estudios Urbanos y Ambientales. Se puede mencionar también que he contribuido a formar jóvenes investigadores, a los que les he dirigido varias decenas de tesis.

Por todo lo anterior me siento bienaventurado por haber tenido la oportunidad de trabajar en el sector académico, el cual me ha permitido desempeñar una labor gratificante que le ha dado sentido a mi vida, más allá del confortable rol de investigador asalariado de clase media. 
INFORMES

\section{Epílogo y agradecimientos: la BUAP, mi quinta alma mater}

En 2010 la población total de México fue de 112 millones de habitantes, de los cuales $70 \%$ son urbanos y habitan en un sistema de 421 ciudades; 11 de éstas son metrópolis con más de un millón de personas, y están encabezadas por: Ciudad de México (19.6), Guadalajara (4.3), Monterrey (3.9), Puebla (2.3) y Toluca (1.7).

Por ende, en los inicios del siglo XxI, México se consolida como nación altamente urbanizada, en donde las ciudades en general, y sus megaurbes en particular, son los escenarios donde se concentran los desequilibrios sociales y urbanísticos. No obstante, éstas constituyen monumentales factores de producción donde se realizan las actividades industriales, comerciales y de servicios, esto es $90 \%$ de la economía, y son indispensables como plataformas para alcanzar un desarrollo sostenido y sustentable.

En el contexto de esta realidad, el H. Consejo Universitario de la Benemérita Universidad Autónoma de Puebla tuvo a bien otorgar a un especialista en la denominada ciencia urbana y regional el grado de Doctor Honoris Causa, incorporándolo a su claustro académico. Ello probablemente muestra la intención de la institución de fomentar y elevar el rigor de la investigación y la docencia en la disciplina urbana dentro de sus planes de promover la excelencia en todas las ramas del conocimiento.

El argumento central de esta intervención sostiene que los investigadores y científicos no surgen por generación espontánea, por mera genética, vocación o virtuosismo personal, sino que son producto de las instituciones de educación universitaria, principalmente las públicas, dentro de una estructura social determinada y de ciertos atributos subjetivos. Ante la pérdida de competitividad educativa y productiva de México dentro de la economía mundial en las últimas tres décadas, raíz de la grave violencia criminal que socava y amenaza con devastar a la república, una de las principales acciones para revertir tan crítica situación es reforzar la calidad académica de las universidades públicas, formando más y mejores investigadores. Ello deberá ser la base para realizar una revisión pormenorizada del modelo económico neoliberal vigente, proponiendo las modificaciones requeridas que hagan posible insertar a la nación dentro de las sociedades del conocimiento del siglo XXI.

Hago público mi reconocimiento a Alfonso Esparza Ortiz, rector de la BUAP, al secretario general, René Valdiviezo Sandoval, a Francis- 
co Vélez Pliego, director del Instituto de Ciencias Sociales y Humanidades (ICSH), quien postuló mi candidatura, así como a mi joven colega Sergio Flores González, por su activa participación en el proceso. Extiendo mi agradecimiento a los integrantes de la Comisión de Grados Honoríficos y Distinciones, así como a todos los miembros del H. Consejo Universitario, quienes aprobaron por unanimidad la propuesta. También quedo en deuda con las instituciones y las personas que, muy pródiga y desinteresadamente, tuvieron a bien apoyarla.

Extiendo mis votos sinceros para que la Benemérita Universidad Autónoma de Puebla, mi nueva alma mater, enfrente consciente y exitosamente el reto que se tiene. Espero tener la oportunidad de contribuir con la universidad, por modesta que pueda ser mi participación, en cuestiones metodológicas y de la praxis de la investigación en economía urbana.

Muchas gracias. 